\title{
SELECCIÓN Y CARACTERIZACIÓN DE CEPAS NATIVAS DE Enterococcus CON POTENCIALIDAD ANTIMICROBIANA AISLADAS DE QUESOS DE ELABORACIÓN ARTESANAL
}

\section{SELECTION AND CHARACTERIZATION OF NATIVE Enterococcus STRAINS WITH ANTIMICROBIAL POTENTIAL ISOLATED FROM ARTISANAL MANUFACTURED CHEESES}

\author{
Elena Quillama Polo ${ }^{1,2}$, Liz Cruz Pio $^{2}$ y Ginger Gandolfo Navarro ${ }^{2}$
}

\begin{abstract}
Resumen
Los Enterococcus constituyen parte de la microbiota autóctona de quesos artesanales y contribuyen con el desarrollo del aroma y maduración de los quesos. Algunas cepas, sintetizan péptidos antimicrobianos denominados enterocinas y pueden suprimir el crecimiento de microorganismos patógenos contaminantes de alimentos. El objetivo, fue seleccionar y caracterizar cepas nativas de Enterococcus con actividad antimicrobiana de quesos artesanales. Para su aislamiento, las muestras fueron sembradas en agar bilis esculina y agar MRS. La caracterización fenotípica así como la detección de sustancias antimicrobianas, fueron realizadas por métodos bioquímicos, bicapa y difusión en agar respectivamente. De 68 cepas aisladas, Enterococcus faecium fue la especie predominante, seguida de Enterococcus durans y Enterococcus faecalis. Asimismo, nueve cepas mostraron actividad antimicrobiana contra bacterias taxonómicamente afines y patógenas contaminantes de alimentos. Las cepas Enterococcus faecium QPa.1 y QPa.2 ejercieron un efecto antagonista muy efectivo frente a Listeria monocytogenes. La sustancia antimicrobiana producida por E. faecium QPa.1 fue inactivada por tripsina y $\alpha$-quimotripsina, pero no por catalasa. Además, dicha actividad fue estable a pH 3 y 9 , a $100{ }^{\circ} \mathrm{C}$ por 20 min y a 4 y $-20{ }^{\circ} \mathrm{C}$ durante 180 días. En conclusión, Enterococcus faecium QPa.1 produjo una bacteriocina activa y específica contra Listeria monocytogenes, con posibilidad de ser utilizada como un cultivo bioprotector para la preservación de quesos.
\end{abstract}

Palabras clave: bacterias lácticas, actividad antimicrobiana, bacteriocinas, quesos.

\begin{abstract}
Enterococcus are part of indigenous microbiota of artisanal cheeses and contribute to the development of the aroma and ripeness of cheeses. Some strains synthesize antimicrobial peptides called enterocins that can suppress the growth of foodborne pathogenic microorganisms. The aim of this research was to select and characterize native strains of Enterococcus with antimicrobial activity isolated from artisanal cheeses. For their isolation, the samples were seeded in bile esculin agar and MRS agar. Phenotypic characterization as well as the detection of antimicrobial substances were carried out by biochemical, bilayer and diffusion on agar methods, respectively. Of 68 strains isolated, Enterococcus faecium was the predominant species, followed by Enterococcus durans and Enterococcus faecalis. Nine strains also showed antimicrobial activity against taxonomically related bacteria and food-borne pathogens. Enterococcus faecium Qpa.1 and Qpa.2 strains exerted a very effective antagonistic effect against Listeria monocytogenes. The antimicrobial substance produced by E. faecium $\mathrm{QPa}$.1 was inactivated by trypsin and $\alpha$-chymotrypsin, but not by catalase. In addition, this activity was stable at $\mathrm{pH} 3$ and 9 , at $100{ }^{\circ} \mathrm{C}$ for 20 min and at 4 and $-20{ }^{\circ} \mathrm{C}$ for 180 days. In conclusion, Enterococcus faecium QPa.1 produced an active and specific bacteriocin against Listeria monocytogenes, which could be used as a bioprotective culture for the preservation of cheeses.
\end{abstract}

Key words: acid lactic bacteria, antimicrobial activity, bacteriocin, cheeses.

\section{Introducción}

La elaboración de quesos se remonta a la época del antiguo Egipto aproximadamente 3007-2975 a. C., alcanzando su popularidad en Grecia y Roma. En 1552, según López de Gomara, fueron los españoles los primeros en introducir el queso al Perú. A partir de esta fecha, su producción se extendió especialmente al sur del país donde adquirió la denominación de JAPCHA o 
KAWCHU, que significa morder jebe o caucho (Velasco del Real, 1892). El queso andino en particular, es un producto tradicional elaborado artesanalmente por los pobladores de la sierra central y sur del Perú. Los quesos son producidos con leche cruda de vaca y/o cabra, sin añadir cultivos iniciadores y son apreciados por sus singulares características sensoriales. En general, los quesos artesanales son obtenidos por coagulación de la leche, separación del suero, salado, moldeado, prensado y en algunos casos pasa por un proceso de maduración, donde ocurren una serie de cambios secuenciales causados por las enzimas coagulantes y fermentos naturales (Sánchez-Ponte, 2003). Estos productos son ricos en proteínas de alta calidad, alto porcentaje de grasa y excelente fuente de calcio, fósforo, hierro y vitaminas. Asimismo, tienen una gran diversidad de sabores, texturas y formas, debido a la variabilidad de leches, las condiciones geográficas, climáticas y técnicas de procesamiento de cada localidad (Beresford et al., 2001).

Los quesos tradicionales, presentan una población microbiana típica y diferente y están conformadas principalmente por bacterias lácticas (BAL) de los géneros: Lactococcus, Lactobacillus, Streptococcus, Leuconostoc y Enterococcus. Algunas cepas, pueden contribuir al desarrollo del aroma y maduración de los quesos, debido a sus actividades proteolítica, lipolítica y capacidad de sintetizar sustancias aromáticas. Además, muchos de sus productos metabólicos como los ácidos orgánicos, diacetilo, peróxido de hidrógeno, reuterina y bacteriocinas, mejoran la calidad y contribuyen a la bioconservación de estos alimentos (Banwo et al., 2013; Medeiros et al., 2016).

Las bacterias lácticas con propiedades probióticas de los géneros Lactobacillus, Bifidobacterium y, recientemente, las del género Enterococcus asociadas a productos lácteos, son utilizadas en alimentos funcionales. El género Enterococcus está conformado por más de 26 especies, se caracterizan por ser ubicuas, habitan los suelos, los alimentos, el agua y el tracto gastrointestinal del hombre y los animales. Algunas cepas benéficas de Enterococcus faecium, E. faecalis y E. durans predominan en productos lácteos (Banwo et al., 2013).

Tradicionalmente, el queso artesanal se produce a partir de leche sin pasteurizar por fermentación espontánea, donde intervienen bacterias lácticas autóctonas. La actividad de las bacterias presentes naturalmente en la leche no es controlada, pudiendo resultar en algunos casos, un producto con características sensoriales menos uniformes (Leroy \& De Vuyst, 2004; Topisirovic et al., 2006; Suskovic et al., 2010). Por lo tanto, la selección de cepas autóctonas de bacterias lácticas con propiedades biotecnológicas es muy importante, para producir quesos con características organolépticas similares al producto artesanal y seguros desde un punto de vista sanitario (Ramírez-López \& Vélez-Ruiz, 2016). De esta manera, se puede mejorar y optimizar el control de los procesos de fermentación y obtener productos con propiedades definidas y constantes (Speranza et al., 2015).

Asimismo, para garantizar la seguridad de los quesos elaborados con leche cruda, se pueden utilizar cepas autóctonas de Enterococcus faecium productoras de bacteriocinas o sus metabolitos, para eliminar el crecimiento de bacterias Gram positivas patógenas contaminantes de alimentos, particularmente Listeria monocytogenes especie muy frecuente en quesos. (Ennahar \& Deschamps, 2000; Tulini et al., 2011; Aspri et al., 2017).

Actualmente, las bacteriocinas producidas por algunas cepas de Enterococcus son usadas en la conservación de un amplio rango de productos alimenticios y son consideradas como una alternativa prometedora para combatir la resistencia antimicrobiana emergente (Hanchi et al., 2018).

El objetivo de este estudio fue seleccionar y caracterizar cepas autóctonas de Enterococcus con actividad antimicrobiana aisladas de quesos regionales de Perú.

\section{Materiales y métodos}

\section{Aislamiento de cepas de Enterococcus}

Un total de 14 muestras de quesos artesanales, procedentes de las localidades de Coracora (Ayacucho), Juliaca y Puno (Puno), Chalhuanca y Soraya (Apurímac), Huancayo, Huánuco, Huancavelica, Cajamarca, Arequipa y Huarochirí (Lima), fueron recolectadas en recipientes estériles y transportadas en condiciones asépticas para su procesamiento inmediato. Para el aislamiento, se tomaron $10 \mathrm{~g}$ de queso y se homogenizaron en $90 \mathrm{ml}$ de solución salina peptonada. Del homogenizado resultante se realizaron diluciones decimales seriadas en solución salina al $0.85 \%$. Luego fueron sembradas por el método de diseminación en agar Man Rogosa Sharpe (MRS) (Merck, Darmstadt, Germany) conteniendo cicloheximida al $0.05 \%$ y agar bilis esculina $\mathrm{pH}$ 6.5. Las placas se incubaron en condiciones de microaerofilia a $30{ }^{\circ} \mathrm{C}$ por $48-72 \mathrm{~h}$. La identificación presuntiva se realizó en base a la caracterización morfológica, comportamiento en medios líquidos y prueba de la catalasa. Los cultivos puros se conservaron a $4{ }^{\circ} \mathrm{C}$ en caldo MRS.

Caracterización fenotípica de cepas de Enterococcus

La identificación bioquímica se realizó según De Vos et al. (2009), utilizando el medio base MRS suplementado con púrpura de bromocresol $\mathrm{pH} 6.5$ conteniendo diversos carbohidratos: arabinosa, glucosa, lactosa, galactosa, maltosa, manitol, sacarosa, ribosa, rafinosa, xilosa y melibiosa al $1 \%$, incluyendo las pruebas de gluconato de potasio, Voges Proskauer, crecimiento en medios líquidos a diferentes temperaturas $\left(37,45\right.$ y $\left.60{ }^{\circ} \mathrm{C}\right)$ durante 15 y $30 \mathrm{~min}$, crecimiento en presencia de $6.5 \% \mathrm{NaCl}, \mathrm{pH} 9.6,40 \%$ de sales biliares e hidrólisis de esculina. 
$\underline{\text { Búsqueda de cepas productoras de sustancias }}$ antagonistas

La exploración de cepas de Enterococcus productoras de sustancias antimicrobianas y cepas sensibles o indicadoras a los compuestos en mención, se realizó mediante la técnica de la bicapa (Barefoot \& Klaenhammer, 1983), enfrentándolas unas contra otras en grupos de 12. Las posibles cepas productoras fueron sembradas por moteado en la superficie de Agar MRS pH 6.5 e incubadas a $30{ }^{\circ} \mathrm{C}$ en condiciones de microaerofilia durante $20 \mathrm{~h}$ hasta obtener un crecimiento visible. Luego, se aplicó una segunda capa de agar MRS semisólido a $50{ }^{\circ} \mathrm{C}$ previamente inoculado con $100 \mu \mathrm{l}$ de un cultivo activo de la posible cepa sensible de $12 \mathrm{~h}$ de crecimiento. Una vez solidificada la bicapa se procedió a incubar a $30{ }^{\circ} \mathrm{C}$ por $18 \mathrm{~h}$ en condiciones de microaerofilia. En esta etapa, no fue excluido el posible efecto inhibitorio de los ácidos orgánicos ni del peróxido de hidrógeno. Las cepas de Enterococcus que mostraron mayores tamaños de halos de inhibición y las cepas sensibles a los compuestos inhibitorios, fueron seleccionadas para la caracterización complementaria.

Caracterización de las sustancias antimicrobianas producidas por las cepas Enterococcus faecium QPa.1 y Enterococcus faecium QPa.2

La caracterización se realizó mediante el método de difusión en agar (Tag \& Mcgiven, 1971) utilizando los sobrenadantes crudos libres de células (SLC) de cultivos activos de las cepas productoras en fase logarítmica tardía y cultivos puros de las cepas indicadoras en fase logarítmica temprana a una D.O $560 \mathrm{~nm}=0.2$. Los SLC fueron filtrados en membrana Millex ${ }^{\circledR}$ GP de $0.22 \mu \mathrm{m}$ (Merck, Darmstadt, Germany) y fraccionados de la siguiente manera: A) Sobrenadante crudo, sin ningún tratamiento (Control), B) Sobrenadante tratado con $\mathrm{NaOH}$ a $\mathrm{pH} \quad 6.5$ (sobrenadante neutralizado), para comprobar si el efecto inhibitorio es debido a la acción de ácidos orgánicos y C) Sobrenadante neutralizado + catalasa (1 $\mathrm{mg} / \mathrm{ml}$; Sigma Aldrich) para demostrar si el efecto inhibitorio del sobrenadante neutralizado es debido a la acción del peróxido de hidrógeno. Seguidamente, los sobrenadantes tratados con antelación, fueron adicionados a los respectivos orificios realizados en agar MRS semisólido conteniendo $100 \mu \mathrm{l}$ de la cepa indicadora Enterococcus faecium QPe.1. Después de 18 h de incubación a $30^{\circ} \mathrm{C}$, se seleccionó la cepa que mostró mayor halo de inhibición frente a la cepa indicadora en mención.

Efecto de las enzimas, temperatura y $\mathrm{pH}$ sobre la $\underline{\text { sustancia antibacteriana producida por Enterococcus }}$ faecium QPa.1

Para comprobar la estabilidad de la sustancia antimicrobiana, el sobrenadante neutralizado libre de células (SNLC) de la cepa seleccionada, fue tratado con tripsina $\mathrm{pH} 6.9$ (Sigma Aldrich) y $\alpha$-quimotripsina $\mathrm{pH}$ 8 (Sigma Aldrich) a una concentración final de $1 \mathrm{mg} / \mathrm{ml}$ e incubado a $30{ }^{\circ} \mathrm{C}$ por dos horas. El SNLC fue también calentado a 80 y $100{ }^{\circ} \mathrm{C}$ por $10 \mathrm{~min}$, a $121{ }^{\circ} \mathrm{C}$ por 15 min y mantenido a 4 y $-20{ }^{\circ} \mathrm{C}$ por 180 días. Asimismo, fue ajustado a pH 3 y 9 . La estabilidad de la sustancia antimicrobiana, fue verificada utilizando el método de difusión en agar.

Determinación del espectro antimicrobiano

La determinación de la capacidad de antibiosis de las cepas Enterococcus faecium QPa.1 y Enterococcus faecium QPa.2 contra bacterias patógenas contaminantes de alimentos: Salmonella typhimurium, Escherichia coli, Bacillus cereus, Listeria monocytogenes y Staphylococcus aureus obtenidas del banco de Cultivos Iniciadores y Probióticos Lácticos, Facultad de Ciencias Biológicas - Universidad Nacional Mayor de San Marcos (CIPROLAC, FCBUNMSM), se realizó por el método de difusión en agar, para lo cual, se prepararon 10 placas con Agar Brain Heart Infussion (BHI) semisólido conteniendo $100 \mu \mathrm{l}$ de cultivos activos de cada una de las cepas patógenas de 12 horas de crecimiento $(\mathrm{D} . \mathrm{O}=0.2$ a $560 \mathrm{~nm}$ ), donde se hicieron los orificios respectivos y se agregaron 100 $\mu \mathrm{l}$ de los sobrenadantes libres de células A, B, C y sobrenadantes ajustados a $\mathrm{pH} 3, \mathrm{pH} 8$ y conservados a $-20{ }^{\circ} \mathrm{C}$ por 180 días. Después de 24 h de incubación, se observaron zonas claras alrededor de los orificios. El caldo MRS sin microorganismo fue utilizado como control negativo.

\section{Resultados y discusión}

Aislamiento y caracterización fenotípica de cepas de Enterococcus

De un total de 14 muestras de quesos frescos y semimaduros de elaboración artesanal procedentes de diferentes zonas del centro y sur de Perú, se lograron aislar e identificar por métodos fenotípicos 68 cepas de Enterococcus (Tabla 1), correspondiendo el 56\% a Enterococcus faecium, el 3\% a Enterococcus durans y el $1 \%$ a Enterococcus faecalis. 27 cepas de Enterococcus no fueron identificadas porque mostraron un perfil bioquímico atípico (Tabla 2). Estas bacterias fueron caracterizadas como cocos Gram positivos, catalasa negativos, tolerantes a altas concentraciones de cloruro de sodio, de sales biliares y a pH 9.6. Además, mostraron resistencia a $60{ }^{\circ} \mathrm{C}$ por 30 min e hidrolizaron la esculina. Estos resultados revelaron que en la mayoría de los quesos evaluados de distinta procedencia, predominó Enterococcus faecium, lo que indica su activa participación en la fermentación de los quesos artesanales. Resultados similares reportaron Jurkovič et al. (2006) y Slyvka et al. (2018) quienes también identificaron las especies de Enterococcus faecium, E. durans y E. faecalis, en quesos bryndza y quesos Carpatianos tradicionales de Eslovaquia, mediante métodos fenotípicos y moleculares. Asimismo, en quesos de elaboración artesanal producidos en dos regiones de Brazil, Medeiros et al. (2016) y Luiz et al. (2016), revelaron la presencia de 
una microbiota diversificada de bacterias lácticas, prevaleciendo las especies Enterococcus faecium, E. faecalis, E. italicus y E. durans, coincidiendo con los resultados obtenidos en este estudio a excepción de Enterococcus italicus.

$\underline{\text { Selección de cepas nativas de Enterococcus con }}$ actividad antimicrobiana

De 68 cepas de Enterococcus aisladas de quesos, se seleccionaron nueve cepas con actividad antimicrobiana por el método de la bicapa (Barefoot \& Klaenhammer, 1983), correspondiendo siete a Enterococcus faecium, una a Enterococcus faecalis y una a Enterococcus durans. Además, se registró un alto porcentaje de cepas sensibles taxonómicamente afines (Tabla 3). Las cepas que mostraron mayores tamaños de halos de inhibición (19 a $20 \mathrm{~mm}$ ) frente a la cepa indicadora QPe.1 fueron Enterococcus faecium QPa.1 y Enterococcus faecium QPa.2 (Figura 1). Otros autores como Franz et al. (1996), emplearon también el método de la bicapa para explorar cepas de Enterococcus con actividad antagonista en productos vegetales fermentados como las aceitunas, utilizando como cepas indicadoras, las especies Lactobacillus sakei DSM 20017, Leuconostoc mesenteroides DSM 20343, Lb. plantarum DSM 20174 y Enterococcus faecalis DSM 20380.

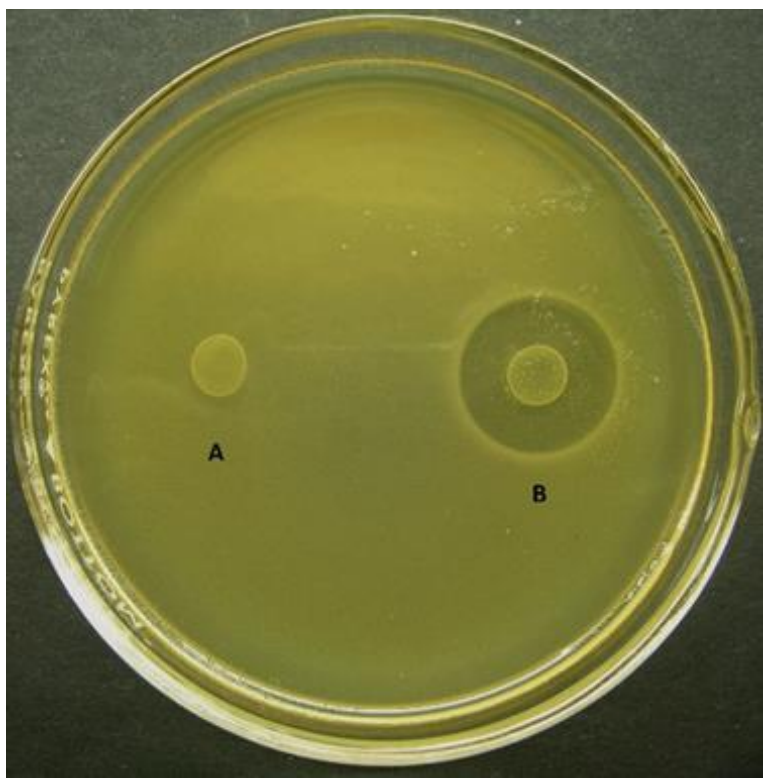

A: Halo de inhibición (negativo) de Lactobacillus casei y B: Halo de inhibición (positivo) de Enterococcus faecium QPa.1 frente a Enterococcus faecium QPe.1.

Figura 1. Actividad antimicrobiana de cepas de bacterias lácticas contra Enterococcus faecium QPe.1.

\section{Caracterización del compuesto inhibitorio producido por las cepas Enterococcus faecium QPa.1 y Enterococcus faecium QPa.2}

Para caracterizar las sustancias antimicrobianas contenidas en los SNLC de Enterococcus faecium
QPa.1 y Enterococcus faecium QPa.2 contra cepas taxonómicamente afines, se utilizó el método de difusión en agar. Los resultados obtenidos indicaron que el efecto de las sustancias antimicrobianas producidas por las cepas productoras, no fue debido a la presencia de ácidos orgánicos ni agua oxigenada (Tabla 4). Resultados coincidentes fueron obtenidos por Khay et al. (2011), quienes seleccionaron a partir de leche de camello en Marruecos, una cepa de Enterococcus faecium R111 y dos cepas de Enterococcus durans E204, y E214 con actividad antimicrobiana contra cepas taxonómicamente afines por el método de difusión en agar.

Determinación del espectro antimicrobiano

Las cepas Enterococcus faecium QPa.1 y Enterococcus faecium QPa.2, aisladas de quesos de elaboración artesanal de la región de Puno, mostraron una actividad inhibitoria efectiva contra E. faecium QPe.1 y Listeria monocytogenes (Tablas 4 y 6, Figura $2)$. Diversos autores también reportaron que ciertas cepas de E. faecium asociados a leche de vaca (Banwo et al., 2013), quesos Koopeh de Iran (Hassanzadazar et al., 2014), quesos Coalho de Brasil (Freitas et al., 2013; Dos Santos et al., 2014) y quesos de cabra de Francia (Hammi et al., 2018), produjeron un efecto inhibitorio contra Listeria monocytogenes, con probabilidades de ser utilizados como probióticos y/o cultivos iniciadores (Banwo et al., 2013; Hassanzadazar et al., 2014; Furlaneto-Maia et al., 2017).

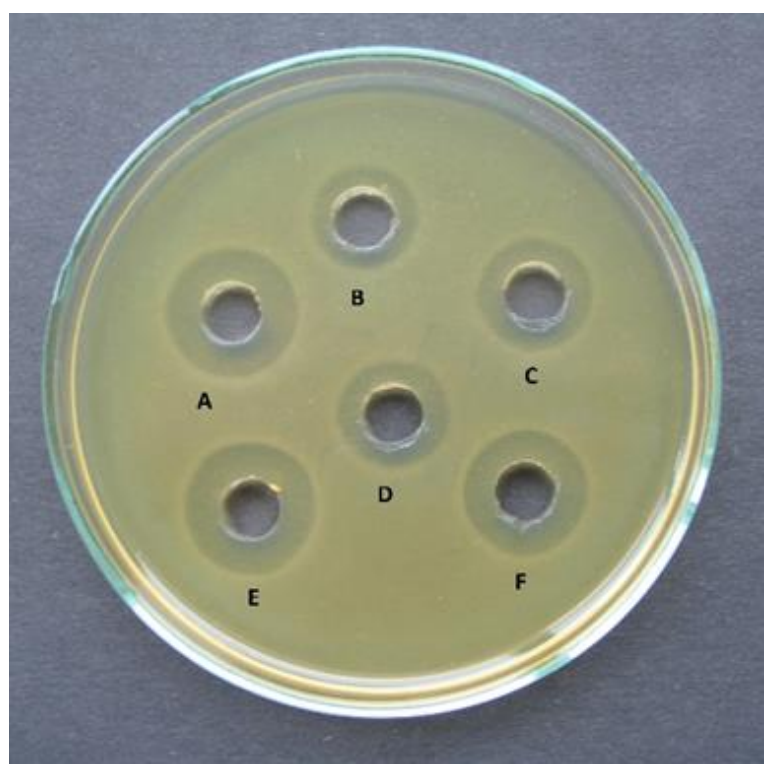

A: Sobrenadante crudo, B: Sobrenadante tratado a $\mathrm{pH}$ 3, C: Sobrenadante tratado a $\mathrm{pH}$ 8, D: Sobrenadante almacenado a $-20^{\circ} \mathrm{C}$ por 180 días, E: Sobrenadante neutralizado y $\mathbf{F}$ : Sobrenadante neutralizado más catalasa.

Figura 2. Actividad inhibitoria de Enterococcus faecium QPa.1 frente a Listeria monocytogenes. 
Efecto de las enzimas, temperatura y $\mathrm{pH}$ sobre la sustancia antibacteriana producida por Enterococcus faecium QPa.1

La actividad antimicrobiana del sobrenadante neutralizado libre de células de la cepa seleccionada $E$. faecium QPa.1 fue completamente inactivada después de ser tratada con las enzimas proteolíticas tripsina y $\alpha$ quimotripsina, indicando que el compuesto inhibitorio es de naturaleza proteica (Tabla 5). Asimismo, cuando el sobrenadante neutralizado libre de células de $E$. faecium QPa.1 fue tratado a diferentes temperaturas, la actividad inhibitoria fue completamente estable a 80 y $100{ }^{\circ} \mathrm{C}$ durante $20 \mathrm{~min}$. Por otro lado, se pudo observar que la actividad de la sustancia antagonista fue también estable a pH 3 y 9 después de 24 horas de incubación a $30{ }^{\circ} \mathrm{C}$. Con respecto a la estabilidad de la sustancia antimicrobiana producida por E. faecium QPa.1 a diferentes temperaturas en función del tiempo, se comprobó que la actividad del SNLC de la cepa productora, fue completamente estable durante 180 días a 4 y $-20{ }^{\circ} \mathrm{C}$.

En base a estos resultados, se confirmó que la sustancia antimicrobiana producida por la cepa Enterococcus faecium QPa.1 es una posible bacteriocina, coincidiendo con los resultados obtenidos por Ennahar \& Deschamps (2000) quienes seleccionaron a partir de queso, la cepa $E$. faecium EF01 de espectro reducido y alta especificidad contra cepas de Listeria monocytogenes y estable a $\mathrm{pH} 4$ hasta 9. Por otro lado, Dos Santos et al. (2014) también demostraron que las sustancias antimicrobianas producidas por E. faecium EM485 y E. faecium EM925, aisladas de quesos de Brasil, fueron inactivadas por enzimas proteolíticas y se mantuvieron estables a pH 2 y 10 , y a $100{ }^{\circ} \mathrm{C}$ por 20 min. También, Banwo et al. (2013) revelaron que las sustancias antagonistas producidas por E. faecium CM4 y E. faecium 2CM1 fueron inactivadas completamente por proteinasa $\mathrm{K}$ y pronasa $\mathrm{E}$, y parcialmente por tripsina. Asimismo, fueron estables a $\mathrm{pH} 4$ y 9 , y mostraron una estabilidad relativa hasta $60{ }^{\circ} \mathrm{C}$ por $30 \mathrm{~min}$ y a $100{ }^{\circ} \mathrm{C}$ por $10 \mathrm{~min}$. La estabilidad térmica es una propiedad común de las bacteriocinas producidas por Enterococcus, y es un rasgo muy importante para su uso como conservante alimentario, ya que muchos procedimientos de procesamiento de alimentos implican un tratamiento térmico (Lee et al., 1999; Yildirim et al., 2014). Franz et al. (1996), comprobaron también que la cepa Enterococcus faecium BFE 900 asociada a aceitunas negras, producía una bacteriocina denominada enterocina 900 , estable a $121{ }^{\circ} \mathrm{C}$ por 15 min y a pH 2 a 10 . A su vez, fue inactivada por pepsina, $\alpha$-quimotripsina, proteinasa $\mathrm{K}$ y tripsina. De la misma manera, Yusuf \& Hamind (2012) demostraron que la actividad de la bacteriocina BFE 900 producida por $E$. faecium B3L3 fue estable a $100{ }^{\circ} \mathrm{C}$ por $15 \mathrm{~min}$, a $4 \mathrm{y}$ $20{ }^{\circ} \mathrm{C}$, a un $\mathrm{pH} 4$ y 10 , y fue completamente inactivada por la tripsina. Tulini et al. (2011) y Aspri et al. (2017) también, han demostrado que las bacteriocinas producidas por las cepas de Enterococcus faecium fueron inactivadas por enzimas proteolíticas, y cuando fueron tratadas a diferentes rangos de $\mathrm{pH} 2$ a 10 y a temperaturas de $121{ }^{\circ} \mathrm{C}$ por $15 \mathrm{~min}$ no perdieron su actividad antimicrobiana. Estos resultados fueron similares para la mayoría de las enterocinas producidas por diferentes cepas nativas de Enterococcus descritas por los autores antes mencionados, incluyendo Enterococcus faecium QPa.1.

La importancia de las bacteriocinas enterococales, radica en su actividad antilisterial. Numerosas cepas de Enterococcus faecium y E. faecalis aisladas de productos lácteos y otras fuentes alimenticias, producen una variedad de sustancias antimicrobianas como las enterocinas con actividad antagonista específica contra Listeria monocytogenes, microorganismo patógeno contaminante de alimentos (Hanchi et al., 2018).

\section{Conclusión}

Nuestro estudio confirmó la presencia de cepas de Enterococcus, como parte importante de la microbiota autóctona de los quesos tradicionales, siendo Enterococcus faecium la especie predominante.

Además, se comprobó que Enterococcus faecium QPa.1 produjo una sustancia antimicrobiana específica contra Listeria monocytogenes, patógeno frecuente en quesos. A su vez, la naturaleza de la sustancia antagonista producida por la cepa en mención no es ácido orgánico ni peróxido de hidrógeno sino una posible bacteriocina, con perspectivas de ser utilizada como biopreservante natural en la elaboración de quesos andinos y otros alimentos regionales.

\section{Agradecimientos}

Al Vicerrectorado de Investigación y al Posgrado de la Universidad Nacional Mayor de San Marcos por el financiamiento.

\section{Literatura citada}

Aspri M., O'Connor P.M., Field D., Cotter P.D., Ross P., Hill C. \& Papademas P. 2017. Application of bacteriocinproducing Enterococcus faecium isolated from donkey milk, in the bio-control of Listeria monocytogenes in fresh whey cheese. International Dairy Journal, 73: 1-9. DOI: 10.1016/j.idairyj.2017.04.008.

Banwo K., Sanni A. \& Tan H. 2013. Technological properties and probiotic potential of Enterococcus faecium strains isolated from cow milk. Journal of Applied Microbiology, 14(1): 229-241. DOI: 10.1111/jam.12031.

Barefoot S.F. \& Klaenhammer T.R. 1983. Detection and activity of lactacin B, a bacteriocin produced by Lactobacillus acidophilus. Applied and Environmental Microbiology, 45(6): 1808-1815. URI: https://aem.asm.org/content/45/6/1808; https://aem.asm.org/content/aem/45/6/1808.full.pdf. 
Beresford T.P., Fitzsimons N.A., Brennan N.L. \& Cogan T.M. 2001. Recent advances in cheese microbiology. International Dairy Journal, 11(4-7): 259-274. DOI: 10.1016/S0958-6946(01)00056-5.

De Vos P., Garrity G., Jones D., Krieg N.R., Ludwig W., Rainey F.A., Schleifer K.H. \& Whitman W.B. (Eds). 2009. Bergey's Manual of Systematic Bacteriology: Volume 3: The Firmicutes. 2nd ed. Springer (Dordrecht, Heidelberg, London and New York). DOI: $10.1007 / \mathrm{b} 92997$.

Dos Santos K.M.O., Vieira A.D.S., Rocha C.R.C., do Nascimento J.C.F., de Souza Lopes A.C., Bruno L.M., Carvalho J.D.G., Gombossy de Melo Franco B.D. \& Todorov S.D. 2014. Brazilian artisanal cheeses as a source of beneficial Enterococcus faecium strains: characterization of the bacteriocinogenic potential. Annals of Microbiology, 64(4): 1463-1471. DOI: 10.1007/s13213-013-0789-4.

Ennahar S. \& Deschamps N. 2000. Anti-Listeria effect of enterocin A, produced by cheese-isolated Enterococcus faecium EFM01, relative to other bacteriocins from lactic acid bacteria. Journal of Applied Microbiology, 88: 449457. DOI: 10.1046/j.1365-2672.2000.00985.x.

Franz C.M.A.P., Schillinger U. \& Holzapfel W.H. 1996. Production and characterization of enterococin 900, a bacteriocin produced by Enterococcus faecium BFE900 from black olives. International Journal of Food Microbiology, 29(2-3): 255-270. DOI: 10.1016/01681605(95)00036-4.

Freitas W.C., Travassos A.E.R. \& Maciel J.F. 2013 Characterization of lactic acid bacteria in coalho cheese and antagonism to some pathogenic food-related bacteria. Revista del Instituto Adolfo Lutz, 72(2): 131-137. URI: http://www.ial.sp.gov.br/resources/insituto-adolfolutz/publicacoes/rial/10/rial72_2_completa/arquivosseparados/1553.pdf.

Furlaneto-Maia L.F., Giazzi A., Brandalize C., Katsuda M.S. Rocha K.R., Terra M.R. \& Furlaneto M.C. 2017. Isolation and characterization of potential probiotic enterococci strains from soft cheese flora. African Journal of Microbiology Research, 11(12): 482-487. DOI: 10.5897/AJMR2017.8429.

Hammi I., Amensag K., Ennahar S., Delalande F., Marchioni E., Cianferani S. \& Belkhou R. 2018. Native production of pediocin PA-1 by Enterococcus faecium E16 isolated from goats' cheese. Journal of Food and Nutrition Research (ISSN 1336-8672), 1:1-8. National Agricultural and Food Centre. Slovakia. URI: http://www.vup.sk/en/download.php?bulID=2005.

Hanchi H., Mottawea W., Sebei K. \& Hammami R. 2018. The Genus Enterococcus: between probiotic potential and safety concerns - an update. Frontiers in Microbiology, 9: Article 1791. DOI: 10.3389/fmicb.2018.01791.

Hassanzadazar H., Ehsani A. \& Mardani K. 2014. Antibacterial activity of Enterococcus faecium derived from Koopeh cheese against Listeria monocytogenes in probiotic ultra-filtrated cheese. Veterinary Research Forum, 5(3): 169-175. URI: http://vrf.iranjournals.ir/article 6620.html.

Jurkovič D., Križková L., Dušinský R., Belicová A., Sojka M., Krajčovič J. \& Ebringer L. 2006. Identification and characterization of enterococci from bryndza cheese. Letters in Applied Microbiology, 42(6): 553-559. DOI: 10.1111/j.1472-765X.2006.01918.x.
Khay E.O., Idaomar M., Pastrana Castro, L.M., Fajardo Bernárdez P., Senhaji N.S. \& Abrini J. 2011. Antimicrobial activities of the bacteriocin-like substances produced by lactic acid bacteria isolated from Moroccan dromedary milk. African Journal of Biotechnology, 10(51): 10447-10455. DOI: 10.5897/AJB11.1328.

Lee H.-J., Joo Y.-J., Park C.-S., Kim S.-H., Hwang I.-K., Ahn J.-S. \& Mheen T.-I.. 1999. Purification and characterization of a bacteriocin produced by Lactococcus lactis subsp. lactis H-559 isolated from kimchi. Journal of Bioscience and Bioengineering, 88(2): 153-159. DOI: 10.1016/s1389-1723(99)80194-7.

Leroy F. \& De Vuyst L. 2004. Lactic acid bacteria as functional starter cultures for the food fermentation industry. Trends Food Sci. Technol., 15(2): 67-78. DOI: https://doi.org/10.1016/j.tifs.2003.09.004.

Luiz L.M.P., Castro R.D., Sandes S.H.C., Silva J.G., Oliveira L.G., Sales G.A., Nunes A.C. \& Souza M.R. 2016. Isolation and identification of lactic acid bacteriafrom Brazilian Minas artisanal cheese. CyTA-Journal of Food, 15(1): 125-128. (vPress: 2017). DOI: 10.1080/19476337.2016.1219392.

Medeiros R.S., Araújo L.M., Queiroga N.V., Andrade P.P., Melo M.A. \& Gonçalves M.M.B.P. 2016. Identification of lactic acid bacteria isolated from artisanal Coalho cheese produced in the Brazilian Northeast. CyTA Journal of Food, 14 (4): 613-620. DOI: 10.1080/19476337.2016.1185468

Pavunc A.L., Beganovi J., Kos B., Uroic K., Blazic M. \& Suskovic J. 2012. Characterization and application of autochthonous starter cultures for fresh cheese production. Food Technology and Biotechnology, 50 (2):141-151.

URI: http://www.ftb.com.hr/images/pdfarticles/2012/AprilJune/141.pdf.

Ramírez-López C. \& Vélez-Ruiz J.F. 2016. Aislamiento, caracterización y selección de bacterias lácticas autóctonas de leche y queso fresco artesanal de cabra. Información Tecnológica, 27(6): 115-128. DOI: 10.4067/S0718-07642016000600012.

Sánchez-Ponte M.D. 2003. Maduración acelerada de queso con bacterias lácticas atenuadas térmicamente. Revista Científica FCV-LUZ, 13(4): 299-306. URI: http://www.produccioncientificaluz.org/index.php/cienti fica/article/view/14991.

Slyvka I.M., Tsisaryk O.Y., Dronyk G.V. \& Musiy L.Y. 2018. Strains of lactic acid bacteria isolated from traditional Carpathian cheeses. Regulatory Mechanisms in Biosystems, 9(1): 62-68. DOI: https://doi.org/10.15421/021808.

Speranza B., Bevilacqua A., Corbo M.R., Altieri C. \& Sinigaglia M. 2015. Selection of autochthonous strains as promising starter cultures for Fior di Latte, a traditional cheese of southern Italy. Journal of Science of Food and Agriculture, 95(1): 88-97. DOI: 10.1002/jsfa.6686.

Suskovic J., Kos B., Beganovic J., Lebos Pavunc A., Habjanic K. \& Matosic S. 2010. Antimicrobial activity The most important property of probiotic and starter lactic acid bacteria. Food Technology Biotechnology, 48(3): 296-307.

URI: https://hrcak.srce.hr/57561. 
Tagg J.R. \& Mcgiven A.R. 1971. Assay System for Bacteriocins. Applied and Environmental Microbiology, 21(5): 943-947. URI: https://aem.asm.org/content/aem/21/5/943.full.pdf.

Topisirovic Lj., Kojic M., Fira Dj., Golic N., Strahinic I. \& Lozo J. 2006. Potential of lactic acid bacteria isolated from specific natural niches in food production and preservation. International Journal of Food Microbiology, 112(3): $230-235$.
10.1016/j.ijfoodmicro.2006.04.009.

ulini F.L., Gomes B.C. \& De Martinis E.C.P. 2011. Partial purification and characterization of a bacteriocin produced by Enterococcus faecium 130 isolated from mozzarella cheese. Ciência e Tecnologia de Alimentos, Campinas, 31(1): 155-159. DOI: 10.1590/S010120612011000100022
Velasco del Real O. 1892. Viaje por la América del Sur: impresiones y recuerdos. $1^{\text {ra }}$ ed. California: R. Molinas.

Yildirim Z., Bilgin H., Isleroglu H., Tokalti K., Sahingil D. \& Yildirim M. 2014. Enterocin HZ produced by a wild Enterococcus faecium strain isolated from a traditional, starter-free pickled cheese. Journal of Dairy Research, 81(2): 164-172. DOI: 10.1017/S0022029914000016.

Yusuf M.A. \& Hamid T.A.T.A. 2012. Optimization of temperature and $\mathrm{pH}$ for the growth and bacteriocina production of Enterococcus faecium. B3L3. IOSR Journal of Pharmacy, 2(6(5)): 49-59. DOI: 10.9790/301326504959.

Tabla 1. Aislamiento de cepas de Enterococcus asociadas a quesos artesanales procedentes de diferentes departamentos del Perú.

\begin{tabular}{|c|c|c|c|c|}
\hline Procedencia & Localidad & $\begin{array}{l}\text { Tipo de } \\
\text { muestra }\end{array}$ & $\begin{array}{l}\text { Tiempo de } \\
\text { maduración (días) }\end{array}$ & Especies \\
\hline \multirow[t]{3}{*}{ Apurímac } & Chalhuanca & fresco & 7 & $\begin{array}{l}\text { Enterococcus sp. (8) } \\
\text { Enterococcus faecium (5) }\end{array}$ \\
\hline & Soraya & maduro & 30 & Enterococcus sp. (1) \\
\hline & Cajamarca & fresco & 7 & Enterococcus sp. (1) \\
\hline Cajamarca & & semimaduro & $15-21$ & $\begin{array}{l}\text { Enterococcus faecium (3) } \\
\text { Enterococcus faecium (1) }\end{array}$ \\
\hline \multirow{4}{*}{$\begin{array}{l}\text { Lima } \\
\text { Arequipa }\end{array}$} & Huarochirí & fresco & 7 & Enterococcus faecium (4) \\
\hline & Yuta & fresco & 7 & $\begin{array}{l}\text { Enterococcus faecium (4) } \\
\text { Enterococcus faecalis (1) }\end{array}$ \\
\hline & Pampa Colca & semimaduro & $15-21$ & Enterococcus sp. (2) \\
\hline & Arequipa & semimaduro & $15-21$ & $\begin{array}{l}\text { Enterococcus faecium (3) } \\
\text { Enterococcus sp. (3) } \\
\text { Enterococcus durans (1) }\end{array}$ \\
\hline \multirow[t]{2}{*}{ Puno } & Puno & semimaduro & $15-21$ & $\begin{array}{l}\text { Enterococcus } \mathrm{sp} .(1) \\
\text { Enterococcus faecium (13) } \\
\text { Enterococcus durans }(1)\end{array}$ \\
\hline & Juliaca & semimaduro & $15-21$ & Enterococcus sp. (2) \\
\hline \multirow{3}{*}{$\begin{array}{l}\text { Huancayo } \\
\text { Huánuco } \\
\text { Ayacucho }\end{array}$} & Huancayo & semimaduro & $15-21$ & Enterococcus faecium (2) \\
\hline & Huánuco & semimaduro & $15-21$ & Enterococcus sp. (4) \\
\hline & Cora Cora & $\begin{array}{l}\text { semimaduro } \\
\text { semimaduro }\end{array}$ & $\begin{array}{c}15-21 \\
30\end{array}$ & $\begin{array}{l}\text { Enterococcus sp. (4) Enterococcus faecium (2) } \\
\text { Enterococcus sp. (1) }\end{array}$ \\
\hline
\end{tabular}


Tabla 2. Caracterización morfológica, cultural, fisiológica y bioquímica de cepas nativas de Enterococcus aisladas de quesos regionales.

\begin{tabular}{|c|c|c|c|c|}
\hline Características & $\begin{array}{c}\text { Enterococcus faecium } \\
\text { (38 cepas) }\end{array}$ & $\begin{array}{l}\text { Enterococcus durans } \\
\text { ( } 2 \text { cepas })\end{array}$ & $\begin{array}{c}\text { Enterococcus faecalis } \\
\text { (1 cepa) }\end{array}$ & $\begin{array}{l}\text { Enterococcus sp. } \\
\text { (27 cepas) }\end{array}$ \\
\hline Morfología de la colonia & $\begin{array}{l}\text { Forma circular, borde } \\
\text { entero, blanca, } 1 \mathrm{~mm} \\
\text { de diámetro }\end{array}$ & $\begin{array}{l}\text { Forma circular, borde } \\
\text { entero, crema, } 1 \mathrm{~mm} \\
\text { de diámetro }\end{array}$ & $\begin{array}{l}\text { Forma circular, borde } \\
\text { entero, } \\
\text { crema, } 1 \mathrm{~mm} \mathrm{de} \\
\text { diámetro }\end{array}$ & $\begin{array}{lr}\text { Forma } & \text { circular, } \\
\text { borde } & \text { entero } \\
\text { amarillo claro }<1 \\
\text { mm de diámetro }\end{array}$ \\
\hline Coloración Gram & $\begin{array}{l}\text { Cocos Gram positivos } \\
\text { en cadenas largas y } \\
\text { cortas }\end{array}$ & $\begin{array}{l}\text { Cocos Gram positivos } \\
\text { en pares y cadenas } \\
\text { cortas }\end{array}$ & $\begin{array}{l}\text { Cocos Gram positivos } \\
\text { en pares y cadenas } \\
\text { cortas }\end{array}$ & $\begin{array}{l}\text { Cocos Gram } \\
\text { positivos en pares, } \\
\text { cadenas cortas }\end{array}$ \\
\hline $\begin{array}{l}\text { Comportamiento en caldo } \\
\text { MRS }\end{array}$ & $\begin{array}{l}\text { Turbidez en } 2 \text { fases, } \\
\text { sedimento } \\
\text { blanquecino compacto }\end{array}$ & $\begin{array}{l}\text { Turbidez homogénea, } \\
\text { sedimento } \\
\text { blanquecino } \\
\text { no compacto }\end{array}$ & $\begin{array}{l}\text { Turbidez en } 2 \text { fases, } \\
\text { sedimento } \\
\text { blanquecino } \\
\text { no compacto }\end{array}$ & $\begin{array}{l}\text { Turbidez en } 2 \\
\text { fases, sedimento } \\
\text { blanquecino } \\
\text { compacto }\end{array}$ \\
\hline $\begin{array}{l}\text { Catalasa } \\
\text { Producción de acidez: }\end{array}$ & - & - & - & - \\
\hline Glucosa & + & + & + & + \\
\hline Lactosa & + & + & + & + \\
\hline Galactosa & + & + & + & + \\
\hline Sacarosa & $\mathrm{v}$ & - & + & + \\
\hline Maltosa & + & + & + & + \\
\hline Manitol & + & - & $\mathrm{d}$ & + \\
\hline Melibiosa & $\mathrm{V}$ & - & - & + \\
\hline Rafinosa & - & - & - & - \\
\hline Ribosa & & & & \\
\hline Arabinosa & $\mathrm{v}$ & - & + & + \\
\hline $\begin{array}{l}\text { Xilosa } \\
\text { Crecimiento: }\end{array}$ & - & - & + & - \\
\hline $45^{\circ} \mathrm{C}$ & + & + & + & + \\
\hline $37^{\circ} \mathrm{C}$ & + & + & + & + \\
\hline pH 9.6 & + & + & + & + \\
\hline $\mathrm{NaCl} 6.5 \%$ & + & + & + & + \\
\hline Sales biliares $40 \%$ & + & + & + & + \\
\hline Sobrevivencia a $60^{\circ} \mathrm{C}$ : & & & & \\
\hline 15 minutos & + & + & + & + \\
\hline 30 minutos & + & + & + & + \\
\hline Voges Proskauer & $\mathrm{v}$ & - & + & - \\
\hline Gluconato de Potasio & $\mathrm{v}$ & + & + & + \\
\hline Hidrólisis de Esculina & + & + & + & + \\
\hline
\end{tabular}

+: Reacción positiva; -: reacción negativa; v: reacción variable 
Tabla 3. Inhibición de cepas taxonómicamente afines por sustancias antimicrobianas producidas por Enterococcus aisladas de quesos regionales.

\begin{tabular}{|c|c|c|}
\hline Cepas productoras & Cepas indicadoras & $\begin{array}{l}\text { Diámetro de inhibición } \\
\qquad(\mathrm{mm})\end{array}$ \\
\hline \multirow[t]{2}{*}{ Enterococcus faecium QHch.3 } & Lactococcus sp. QCS.d & 5.0 \\
\hline & Lactococcus sp. QCCF.c & 2.0 \\
\hline \multirow{13}{*}{ Enterococcus faecalis QYA.b } & Lactococcus lactis QYA.a & 15.0 \\
\hline & Lactococcus lactis QYA.c & 18.0 \\
\hline & Lactococcus lactis QYA.e & 18.0 \\
\hline & Lactococcus lactis QYA.f & 18.0 \\
\hline & Lactococcus lactis QYA.g & 18.0 \\
\hline & Lactococcus lactis QYA.2 & 15.0 \\
\hline & Lactococcus lactis QYA.3 & 14.0 \\
\hline & Lactococcus lactis QYA.4 & 17.0 \\
\hline & Lactococcus lactis QYA.5 & 18.0 \\
\hline & Enterococcus durans QPe & 13.0 \\
\hline & Enterococcus faecium QPf.1 & 12.0 \\
\hline & Enterococcus faecium QP5.1 & 18.0 \\
\hline & Enterococcus faecium QPe. 1 & 15.0 \\
\hline \multirow[t]{5}{*}{ Enterococcus faecium QPa.1 } & Enterococcus faecium $\mathrm{QPb} .1$ & 18.0 \\
\hline & Enterococcus faecium QPf.1 & 13.0 \\
\hline & Enterococcus faecium QP5.1 & 18.0 \\
\hline & Lactobacillus sp. QP5.2a & 18.0 \\
\hline & Enterococcus faecium QPe. 1 & 20.0 \\
\hline \multirow[t]{7}{*}{ Enterococcus faecium $\mathrm{QPa} 2$} & Enterococcus faecium QP8 & 18.0 \\
\hline & Enterococcus faecium QPc.2 & 18.0 \\
\hline & Enterococcus faecium QPe.1 & 20.0 \\
\hline & Enterococcus faecium QPf.1 & 16.0 \\
\hline & Enterococcus durans QPe & 15.0 \\
\hline & Lactococcus lactis QYA.5 & 10.0 \\
\hline & Enterococcus faecium QP5.1 & 18.0 \\
\hline \multirow[t]{5}{*}{ Enterococcus faecium QP7 } & Enterococcus durans QPe & 14.0 \\
\hline & Enterococcus sp. QC2 & 11.0 \\
\hline & Enterococcus faecium QPe.1 & 8.0 \\
\hline & Enterococcus faecium QPf.1 & 7.0 \\
\hline & Enterococcus faecium QP5.1 & 8.0 \\
\hline \multirow[t]{8}{*}{ Enterococcus faecium $\mathrm{QPb} .2$} & Enterococcus faecium QP8 & 16.0 \\
\hline & Enterococcus faecium QPc.2 & 9.0 \\
\hline & Enterococcus faecium QPe.1 & 10.0 \\
\hline & Enterococcus faecium QPf.1 & 7.0 \\
\hline & Enterococcus faecium QP2.2 & 14.0 \\
\hline & Enterococcus durans QPe & 6.0 \\
\hline & Enterococcus faecium QP5.1 & 8.0 \\
\hline & Enterococcus faecium QP8 & 16.0 \\
\hline \multirow[t]{7}{*}{ Enterococcus faecium QP5.2 } & Enterococcus faecium QPc.2 & 8.0 \\
\hline & Enterococcus faecium QPe. 1 & 10.0 \\
\hline & Enterococcus faecium QPf.1 & 9.0 \\
\hline & Enterococcus faecium QP2.2 & 10.0 \\
\hline & Enterococcus durans QPe & 6.0 \\
\hline & Enterococcus faecium QP5.1 & 7.0 \\
\hline & Lactococcus lactis QPA.5 & 9.0 \\
\hline \multirow[t]{2}{*}{ Enterococcus faecium QPA.I } & Lactococcus lactis QPA.d & 9.0 \\
\hline & Enterococcus faecium QP2.2 & 7.0 \\
\hline Enterococcus durans QPA.f & Lactococcus sp. QCCF.c & 12.0 \\
\hline
\end{tabular}


Tabla 4. Actividad antimicrobiana de cepas de Enterococcus faecium contra bacterias estrechamente relacionadas.

\begin{tabular}{llc}
\hline Cepas productoras & Cepas indicadoras & $\begin{array}{c}\text { Diámetro de Inhibición } \\
(\mathrm{mm})\end{array}$ \\
\hline Enterococcus faecium QPa.1 & Enterococcus faecium QPe.1 & 20 \\
& Enterococcus faecium QPb.1 & 18 \\
& Enterococcus faecium QPf.1 & 13 \\
& Enterococcus faecium QP5.1 & 18 \\
\cline { 2 - 3 } Enterococcus faecium QPa.2 & Enterococcus faecium QP8 & 18 \\
& Enterococcus faecium QPc.2 & 18 \\
& Enterococcus faecium QPe.1 & 19 \\
& Enterococcus faecium QPf.1 & 16 \\
& Enterococcus durans QPe & 15 \\
& Lactococcus lactis QY $\mathrm{A.5}$ & 10 \\
& Enterococcus faecium QP5.1 & 18 \\
\hline
\end{tabular}

Tabla 5. Efecto de enzimas sobre el sobrenadante neutralizado de un cultivo de Enterococcus faecium QPa.1.

\begin{tabular}{lc}
\hline Tratamiento enzimático & $\begin{array}{c}\text { Diámetro de actividad } \\
(\mathrm{mm})\end{array}$ \\
\hline Control & 20.0 \\
Tripsina & 0.0 \\
$\alpha$-quimotripsina & 0.0 \\
\hline
\end{tabular}

Tabla 6. Efecto inhibitorio de cepas nativas de Enterococcus faecium contra bacterias patógenas contaminantes de alimentos.

\begin{tabular}{|c|c|c|c|c|c|c|}
\hline \multirow[b]{2}{*}{ Cepa } & \multicolumn{6}{|c|}{ Diámetro de la zona de inhibición $(\mathrm{mm})$ contra cepas sensibles } \\
\hline & 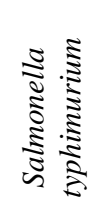 & 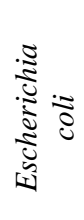 & 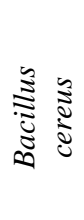 & 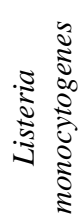 & 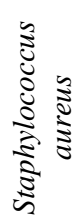 & 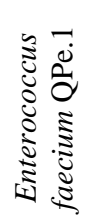 \\
\hline Enterococcus faecium $\mathrm{QPa} .1$ & 4 & 4 & 6 & 20 & 6 & 20 \\
\hline $\begin{array}{l}\text { Enterococcus faecium QPa. } 2 \\
\text { Cepa indicadora: E. faecium }\end{array}$ & 4 & 4 & 4 & 17 & 4 & 19 \\
\hline
\end{tabular}

${ }^{1}$ Autor de correspondencia: equillamap@unmsm.edu.pe.

2 Laboratorio de Microbiología Industrial y Biotecnología Alimentaria. Facultad de Ciencias Biológicas. Universidad Nacional Mayor de San Marcos. Av. Venezuela s/n, Lima 1, Perú. 